\title{
A Fibre-Reinforced Fixed Partial Denture on a Hemisectioned Tooth: A Case Report
}

\author{
Süleyman AGÜLOĞLU ${ }^{1}$, Emrah AYNA ${ }^{2}$, Eylem ÖZDEMİR ${ }^{1}$ \\ ${ }^{1}$ Assistant Professor, Dicle University, Faculty of Dentistry, Department of Prosthetic Dentistry, Diyarbakır, TURKEY \\ ${ }^{2}$ Associate Professor, Dicle University, Faculty of Dentistry, Department of Prosthetic Dentistry, Diyarbakır, TURKEY
}

\section{Key Words}

Fibre-reinforced, adhesive

bridge, hemisection

\author{
Correspondence: \\ Eylem ÖZDEMİR \\ Dicle University, \\ Faculty of Dentistry, \\ Department of Prosthetic Dentistry, \\ 21280, Diyarbakir, TURKEY. \\ e-mail: dteylem@yahoo.com
}

\begin{abstract}
In modern dentistry, fibre-reinforced fixed dental prostheses are considered a useful alternative to classical metal-ceramic restorations. This method allows a conservative approach for replacing missing teeth that overcomes some of the drawbacks of conventional prostheses. Our patient required extraction of tooth \#46 because of an apical lesion of the mesial root, and underwent extraction by hemisection. After healing, using the superior properties of the combined fibre/composite, an adhesive bridge restoration was applied with support from the distal root of tooth \#46 and teeth \#45 and \#47.
\end{abstract}

(Int Dent Res 2011;1:38-41)

\section{Introduction}

For many years, the only prosthetic application used to deal with cases with a single missing tooth was a fixed partial prosthesis. However, the preparation of the two teeth required for correction of a single tooth deficiency causes unnecessary tissue loss. Subsequently, implant-supported fixed prostheses have been developed as an effective solution for this situation. Inevitably, implants cannot be used in some patients because surgical intervention is contraindicated due to systemic disease or the high cost of the operation. Adhesive bridges are a good alternative, as they are less expensive than implants, they do not require surgical intervention and the loss of material from the supporting tooth is small compared to conventional bridges. With the development of fibrestrengthened composites, alternative restorations have become very popular (1-4).

Fibre-reinforced composites have good mechanical properties and their endurance/weight ratios are high compared to metal alloys. Fibrereinforced composites have several advantages: they are translucent, unlike metals; non-corrosive, easy to repair, have good adhesive properties and it is easy to prepare the mouth to receive them. In dentistry, glass, polyethylene and carbon fibres are mainly used as the strengthening materials for composites. Saturated fibres are used as the substructure material for a fixed partial prosthesis, whereas ceramic-reinforced restorative composites are used as the superstructure material (1-7)

\section{Case Report}

A 31-year-old male patient visited our faculty complaining of problems with tooth \#46, and an apical lesion of the mesial root was detected. Based on the results of radiography and intraoral inspections, the distal root of the tooth was deemed usable (Fig. 1A). After endodontic therapy, the tooth was hemisectioned and the mesial root was separated (Fig. 1B and Fig. 1C). Following the healing period, a mandibular impression was obtained and tooth wax was used to plan the bodygum relationship for model preparation (Fig. 1D and Fig. 1E). 


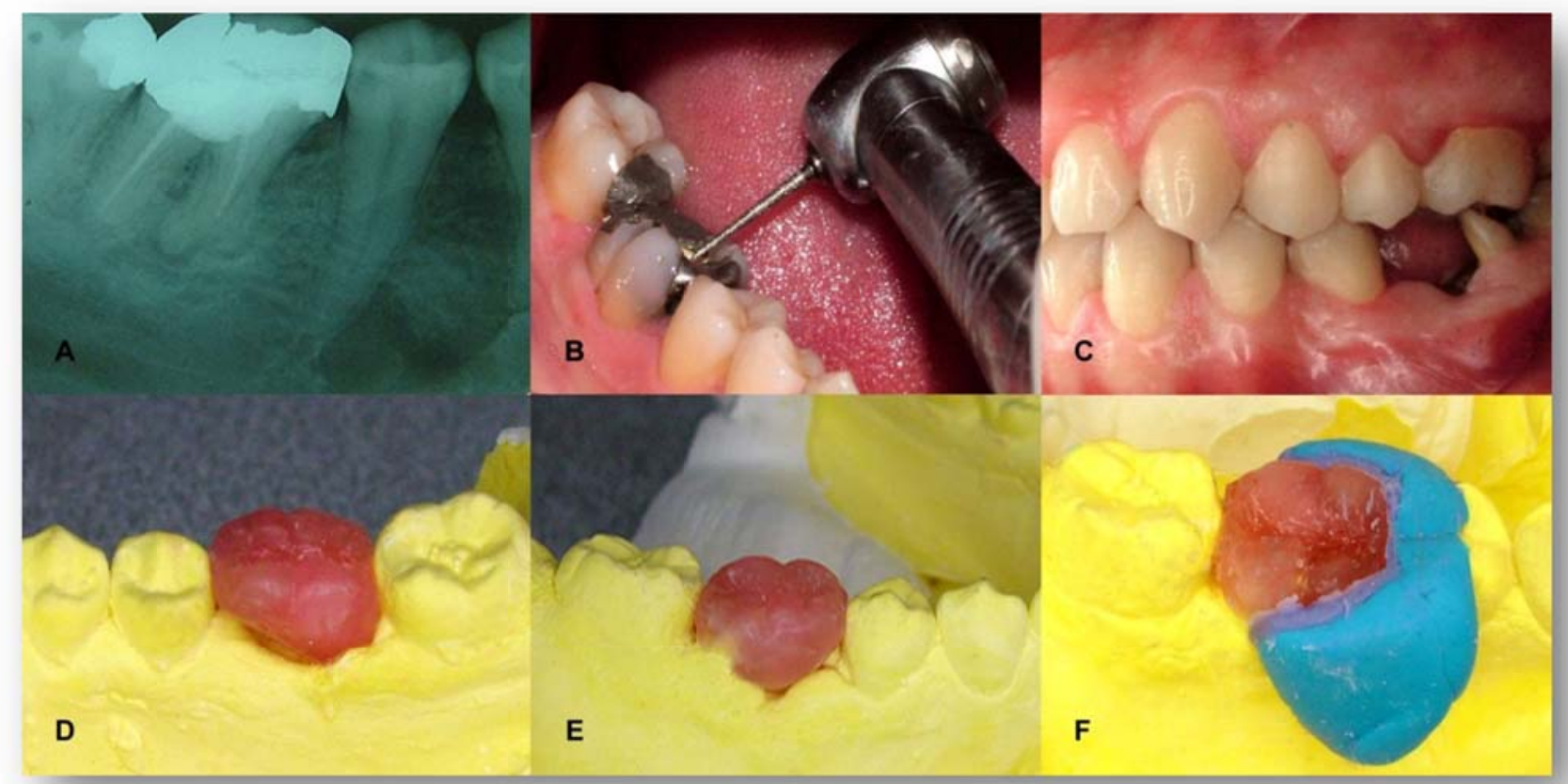

Figure 1. (A)The radiographic image of the tooth \#46; (B) Separating the roots of the tooth \#46; (C) The remaining distal root; (D) The buccal design of the tooth wax that was used to plan the body-gum relationship for model preparation; (E) The lingual design of the tooth wax that was used to plan the bodygum relationship for model preparation; (F) The obtained impression of the tooth wax.

Using a piece of silicon, a sub-body impression of the wax tooth was obtained (Fig. 1F and Fig. 2A). This impression was then placed in the toothless region and the composites were built on top of it as the next step in processing the mouth. On the next visit, a rubber dam was placed in the mouth, and a first glass fibre post (Snowpost; Kuraray Medical, Japan) was placed in the remaining distal root of tooth \#46 (Fig. 2B) for strength, and the crown length was increased. Cavities were prepared for placing polyethylene fibre in teeth \#45 and \#47 and these were etched with 35\% phosphoric acid for 15 seconds after the polyethylene fibre cord (Construct, Kerr Corp., Switzerland) was cut to twice the length of the restoration region, and the cavity was then washed and dried. Clearfil SE Bond primer (Kuraray Medical) was applied for $20 \mathrm{~s}$, and the cavity was dried with slow air flow. To give the ideal shape to the body-gum, silicon previously prepared on top of the model was placed over the crest and then Clearfil SE Bond (Kuraray Medical) was applied to the cavities. In addition, a polyethylene fibre cord previously cut to the appropriate size was wetted with bonding agent and placed as a double layer between the cavities in teeth \#45 and \#47 such that it also adhered to the distal root of \#46 (Fig. 2C). It was then polymerised with light for $10 \mathrm{~s}$. Clearfil Photo Posterior (Kuraray Medical) composite was placed on top of the silicon model former to cover the entire fibre and tooth \#46 was shaped and polymerised with light. The silicon model former was cut and removed from the mouth. Finally, the occlusion of the new tooth was shaped and polished (Fig. 2D and Fig. 2E).

\section{Discussion}

In this study, a glasis fibre post was used to strengthen and lengthen the root. Torbjorner and Fransson reported that crown fabrication using prefabricated posts and resin composite is a viable technique for weakened, endodontically treated roots, especially for the anterior teeth of teenagers in whom a post-core crown is contraindicated (8). Peroz et al. reported that adhesive cementation results in lower microleakage and higher retention and that it is preferred for metal posts (9). 


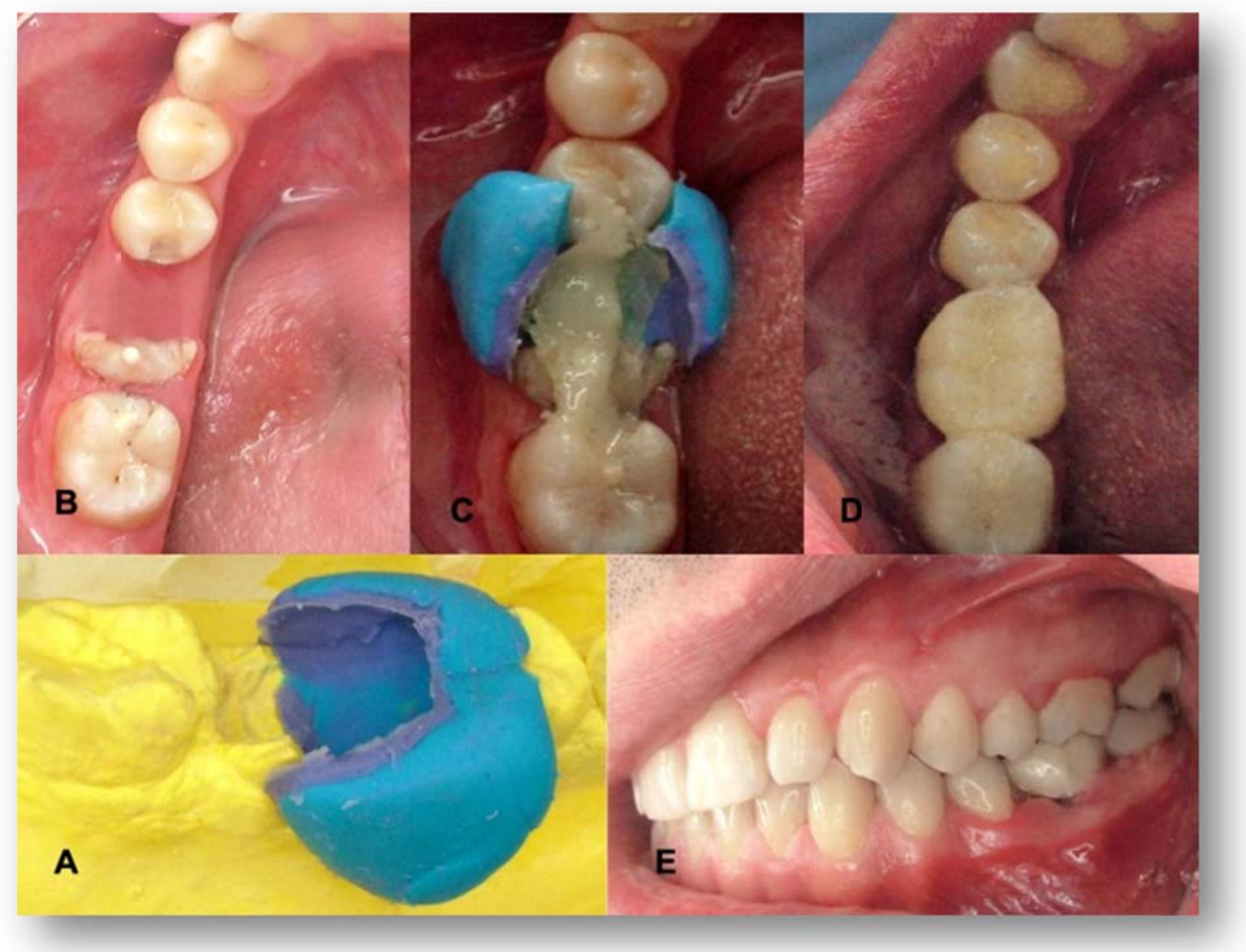

Figure 2. (A) The silicon model former; (B) Glass fibre post was placed in the remaining distal root of tooth \#46; (C) The silicon model former at work; (D) The prepared tooth; (E) The last occlusion and bodygum relationship design.

Previously, adhesive bridges made with fibrereinforced composites were considered temporary restorations. However, with progress in material science, these restorations have been used for many years. Furthermore, these teeth are not as hard as porcelain teeth, which reduces the wear on the opposite tooth. In addition, they are translucent, have good adhesive properties, are easy to prepare and repair, and they are resistant to corrosion. The use of fibre-reinforced composite in resin-bonded fixed partial dentures (FPDs) has been suggested because the composite luting agent adheres better to the framework, the results are good aesthetically and the framework material has physiological stiffness.

The reported 5-year survival rate of metal alloy/resin-bonded FPDs with one pontic is $61 \%$. If the rebonded FPDs in this study were reconsidered in the statistical analysis, the survival rate would increase to $76 \%$ after 5 years, and this has been referred to as the functional survival rate (5). Some studies have indicated that resin-bonded FPDs placed in the mandible have lower survival rates than those placed in the maxilla, while other studies indicated no differences in survival of FPDs located in the mandible and maxilla (2-5). Altieri et al. applied fixed adhesive prostheses with fibrereinforced composite in 14 patients without preparing the tooth and achieved $50 \%$ success after a one-year period (1).

In summary, although the region restored in our patient is a region used for chewing, we believe that the restoration will survive for a long time because of the support from the distal root of tooth \#46, together with the support from teeth \#45 and \#47.

\section{References}

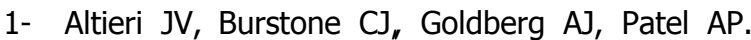
Longitudinal clinical evaluation of fiber-reinforced composite fixed partial dentures: a pilot study. J Prosthet Dent. 1994 Jani;71(1):16-6

2- Mudassir, Y.E. Aboush, M. Hosein, T. Hosein and I. Padihar. Long-term clinical performance of 
resin-bonded fixed partial dentures placed in a developing country. J Prosthodont 1995; 4: 2333

3- P. Rammelsberg, P. Pospiech and W. Gernet. Clinical factors affecting adhesive fixed partial dentures: a 6-year study. J Prosthet Dent. 1993; 70: 300-7

4- M. Behr, A. Leibrock, W. Stich, P. Rammelsberg, M. Rosentritt and G. Handel. Adhesive-fixed partial dentures in anterior and posterior areas. Results of an on-going prospective study begun in 1985. Clin Oral Investig. 1998; 2: 31-4

5- Vallittu PK. Survival rates of resin-bonded, glass fiber-reinforced composite fixed partial dentures with a mean follow-up of 42 months: a pilot study. J Prosthet Dent._2004 Mar; 91(3): 241-6.

6- N.H. Creugers, P.A. Snoek, M.A. van't Hof and A.F. Kayser. Clinical performance of resin-bonded bridges: a 5-year prospective study: II. The influence of patient-dependent variables. J Oral Rehabil 1989; 16: 521-6

7- H.K. Chang, O. Zidan, I.K. Lee and O. GomezMarin. Resin-bonded fixed partial dentures: a recall study. J Prosthet Dent 1991; 65:778-3

8- Torbjorner A, Fransson B. A literature review on the prosthetic treatment of structurally compromised teeth. Int J Prosthodont 2004; 17:369-7

9- Peroz I, Blankenstein F, Lange KP, Naumann M. Restoring endodontically treated teeth with posts and cores: a review. Quintessence Int 2005; 36:737-9 